\title{
Fontan palliation in children is associated with
}

bone deficits

4

TEALTH

University of Minnesota

Masonic Children's Hospital

Anna Petryk ${ }^{1}$, Lynda E. Polgreen², Roland Brown'1, Bradley S. Marino ${ }^{3}$, David Gremmels $^{4}$, Charles Shepard ${ }^{4}$, Aaron S. Kelly ${ }^{1}$, Bradley S. Miller ${ }^{1}$, Kyle Rudser ${ }^{1}$, Lazaros K. Kochilas 5

1 University of Minnesota, Minneapolis, MN; ${ }^{2}$ LA BioMed at Harbor-UCLA, Torrance, CA; ${ }^{3}$ Northwestern University Feinberg

School of Medicine, Ann \& Robert H. Lurie Children's Hospital of Chicago, IL; ${ }^{4}$ The Children's Heart Clinic at Children's

Hospitals and Clinics of Minnesota, Minneapolis, MN; ${ }^{5}$ Emory University and Children's Healthcare of Atlanta, Atlanta, GA, USA

\section{Background}

Survivors with Fontan circulation suffer from chronic systemic hypoperfusion resulting in end-organ injury. Little is known about the effects of these hemodynamic perturbations on bone.

\section{Hypothesis}

Chronic Fontan circulation ( $>5$ years after surgery) is associated with bone deficits.

\section{Methods}

Peripheral quantitative computed tomography (pQCT) was performed on 10 Fontan patients ( 7 males, $11.8 \pm 1.7$ years) and 11 healthy controls (9 males, $12.0 \pm 1.5$ years) with Tanner stage $\leq 3$. Height-adjusted bone mineral density Z-scores for lumbar spine (LBMD) and total body less head (TBLH) were also measured in Fontan patients by dual energy $x$-ray absorptiometry (DXA). Linear regression was used to compare radius $\mathrm{PQCT}$ measures of bone strength index (BSI), trabecular (3\% site) and cortical (33\% site) volumetric BMD (vBMD), thickness, bone mineral content (BMC), cross-sectional area (CSA), polar section modulus (Zp), and strength strain index (SSI) between Fontan patients and controls, and to correlate PQCT outcomes with DXA Z-scores.

\section{Results}

Fontan patients $(\mathrm{N}=10)$ and controls $(\mathrm{N}=11)$ were similar in terms of sex, race, age, pubertal development, and anthropometrics Table 1).

Table 1. Patient characteristics

\begin{tabular}{llll}
\hline & Fontan & Controls & P-value \\
\hline Male & $7(70 \%)$ & $9(82 \%)$ & 0.903 \\
Race/Ethnicity & & & \\
- American Indian & $1(10 \%)$ & $0(0 \%)$ & 0.468 \\
- Black & $1(10 \%)$ & $0(0 \%)$ & \\
- Hispanic/Latino & $1(10 \%)$ & $2(18 \%)$ & \\
- White & $7(70 \%)$ & $9(82 \%)$ & \\
Tanner - Pubic Hair & & & \\
-1 & $4(40 \%)$ & $7(64 \%)$ & 0.556 \\
-2 & $3(30 \%)$ & $2(18 \%)$ & \\
-3 & $3(30 \%)$ & $2(18 \%)$ & \\
Age & $11.8(1.7)$ & $12.0(1.5)$ & 0.737 \\
Height Z-Score & $-0.1(1.3)$ & $-0.6(1.0)$ & 0.317 \\
Weight Z-Score & $-0.1(0.9)$ & $-0.6(0.9)$ & 0.203 \\
BMI Z-Score & $0.0(1.0)$ & $-0.28(0.8)$ & 0.464 \\
\hline Values presented are mean (SD) or N (\%) where indicated. &
\end{tabular}

Fontan patients had lower adjusted means for trabecular vBMD, total vBMD, cortical thickness, and bone strength index (BSI).

Table 2. Linear regression results of adjusted differences in means of Fontan vs. pQCT controls in bone outcomes.

\begin{tabular}{llll}
\hline Fontan & Units & Controls & P-value \\
\hline Trabecular vBMD & $\mathrm{mg} / \mathrm{cm}^{3}$ & $\mathbf{- 2 8 . 1}(-\mathbf{5 4 . 8 , - 1 . 3 )}$ & $\mathbf{0 . 0 4 0}$ \\
Trabecular CSA & $\mathrm{mm}^{2}$ & $25.0(-37.2,87.2)$ & 0.431 \\
Total vBMD & $\mathrm{mg} / \mathrm{cm}^{3}$ & $\mathbf{- 4 1 . 0}(-\mathbf{7 8 . 4 ,}, \mathbf{3 . 6})$ & $\mathbf{0 . 0 3 2}$ \\
Total CSA & $\mathrm{mm}^{2}$ & $-30.4(-99.3,38.4)$ & 0.387 \\
Cortical vBMD & $\mathrm{mg} / \mathrm{cm}^{3}$ & $-10.9(-49.8,28.0)$ & 0.584 \\
Cortical CSA & $\mathrm{mm}^{2}$ & $-6.0(-15.1,3.2)$ & 0.204 \\
Cortical BMC & $\mathrm{mg} / \mathrm{mm}^{2}$ & $-7.0(-17.2,3.2)$ & 0.181 \\
Cortical thickness & $\mathrm{mm}$ & $\mathbf{- 0 . 3 5}(-\mathbf{0 . 6 4}, \mathbf{- 0 . 0 6 )}$ & $\mathbf{0 . 0 1 7}$ \\
Periosteal circumference & $\mathrm{mm}$ & $-0.3(-2.8,2.2)$ & 0.821 \\
Endosteal circumference & $\mathrm{mm}$ & $1.9(-0.7,4.6)$ & 0.153 \\
\hline Total BSI & $\mathrm{mg}^{2} / \mathrm{mm}^{4}$ & $\mathbf{- 7 . 2 ( - 1 3 . 7 , ~ - 0 . 8 )}$ & $\mathbf{0 . 0 2 8}$ \\
Polar Section Modulus (Zp) & $\mathrm{mm}^{3}$ & $-9.3(-51.1,32.6)$ & 0.664 \\
Strength Strain Index (SSI) & $\mathrm{mm}^{3}$ & $-12.6(-47.4,22.3)$ & 0.480 \\
\hline
\end{tabular}

Measures of bone density and geometry were adjusted for radius length, age, and sex, and measure of bone strength were adjusted for age and sex.

Mean height-adjusted LBMD Z-score in Fontan patients was $-0.46 \pm 1.1$ and TBLH Z-score was $-0.63 \pm 1.1$.

- LBMD Z-scores were trending higher by 0.39 per $20 \mathrm{mg} / \mathrm{cm}^{3}$ of trabecular vBMD among Fontan patients.

Table 3. Results of a regression analysis of corrected BMD Z-scores on BMD measurements adjusting for radius length.

\begin{tabular}{llll}
\hline & Covariate & Estimate (95\% Cl) & P-value \\
\hline cLBMD Z-score & $\begin{array}{l}\text { Radius trabecular } \\
\text { BMD (per 20 units) }\end{array}$ & $\begin{array}{l}0.39 \\
(-0.006,0.786)\end{array}$ & 0.054 \\
\hline $\begin{array}{l}\text { CTBLH BMD Z- } \\
\text { score }\end{array}$ & $\begin{array}{l}\text { Radius Cortical } \\
\text { BMD (per 20 units) }\end{array}$ & $\begin{array}{l}0.11 \\
(-0.251,0.466)\end{array}$ & 0.557 \\
\hline
\end{tabular}

DXA data were available for 7 Fontan subjects.

\section{Conclusion}

Children with Fontan palliation have lower trabecular vBMD, total vBMD, cortical thickness, and bone strength index. This suggests that bone metabolism is sensitive to the Fontan physiology. Among pQCT outcomes, trabecular vBMD showed a similar trend as LBMD, underscoring a potential utility of DXA, which is more widely available than pQCT, as a monitoring tool. Further studies should evaluate the etiology and impact on fracture risk of these deficits.

Supported by the NHLBI R01HL110957, the National Center for Advancing Translational Sciences of the NIH Award Number UL1TR000114, NCATS UL1TR000114, Irvine McQuarrie Research Scholar award, and the Children's Heart Foundation. 